\title{
BMJ Open Exploring patients' experiences of analgesia after major lower limb amputation: a qualitative study
}

\author{
Sarah Milosevic (D) , ${ }^{1}$ Heather Strange, ${ }^{1}$ Melanie Morgan, ${ }^{1}$ Graeme K Ambler, ${ }^{2,3}$ \\ David C Bosanquet, ${ }^{4}$ Cherry-Ann Waldron (D) , ${ }^{1}$ Emma Thomas-Jones, ${ }^{1}$ \\ Debbie Harris, ${ }^{1}$ Christopher P Twine, ${ }^{2,3}$ Lucy Brookes-Howell ${ }^{1}$
}

To cite: Milosevic S, Strange $\mathrm{H}$, Morgan $\mathrm{M}$, et al. Exploring patients' experiences of analgesia after major lower limb amputation: a qualitative study. BMJ Open 2021;11:e054618. doi:10.1136/ bmjopen-2021-054618

- Prepublication history for this paper is available online. To view these files, please visit the journal online (http://dx.doi org/10.1136/bmjopen-2021054618).

Received 17 June 2021 Accepted 09 November 2021

Check for updates

(c) Author(s) (or their employer(s)) 2021. Re-use permitted under CC BY-NC. No commercial re-use. See rights and permissions. Published by BMJ.

${ }^{1}$ Centre for Trials Research, Cardiff University, Cardiff, UK ${ }^{2}$ Department of General Surgery, University Hospitals Bristol and Weston NHS Foundation Trust, Bristol, UK

${ }^{3}$ Vascular Department, North Bristol NHS Trust, Bristol, UK

${ }^{4}$ Gwent Vascular Institute,

Royal Gwent Hospital, Aneurin Bevan University Health Board, Newport, UK

Correspondence to

Dr Sarah Milosevic;

milosevics@cardiff.ac.uk

\section{ABSTRACT}

Objectives To explore patient experiences, understanding and perceptions of analgesia following major lower limb amputation.

Design Qualitative interview study, conducted as part of a randomised controlled feasibility trial.

Setting Participants were recruited from two general hospitals in South Wales.

Participants Interview participants were patients enrolled in PLACEMENT (Perineural Local Anaesthetic Catheter aftEr Major lowEr limb amputatioN Trial): a randomised controlled feasibility trial comparing the use of perineural catheter (PNC) versus standard care for postoperative pain relief following major lower limb amputation. PLACEMENT participants who completed 5-day postoperative followup, were able and willing to participate in a face-to-face interview, and had consented to be contacted, were eligible to take part in the qualitative study. A total of 20 interviews were conducted with 14 participants: 10 male and 4 female.

Methods Semi-structured, face-to-face interviews were conducted with participants over two time points: (1) up to 1 month and (2) at least 6 months following amputation. Interviews were audio-recorded, transcribed verbatim and analysed using a framework approach.

Results Interviews revealed unanticipated benefits of PNC usage for postoperative pain relief. Participants valued the localised and continuous nature of this mode of analgesia in comparison to opioids. Concerns about opioid dependence and side effects of pain relief medication were raised by participants in both treatment groups, with some reporting trying to limit their intake of analgesics.

Conclusions Findings suggest routine placement of a PNC following major lower limb amputation could reduce postoperative pain, particularly for patient groups at risk of postoperative delirium. This method of analgesic delivery also has the potential to reduce preoperative anxiety, alleviate the burden of pain management and minimise opioid use. Future research could further examine the comparison between patient-controlled analgesia and continuous analgesia in relation to patient anxiety and satisfaction with pain management.

Trial registration number ISRCTN: 85710690; EudraCT: 2016-003544-37.
Strengths and limitations of this study

- Despite the importance of patient perceptions of pain management, it is believed this is the first study to qualitatively focus on the experiences of patients receiving a perineural catheter to relieve postoperative pain.

- Using qualitative interviews enabled an in-depth understanding of patients' perceptions and experiences of analgesia following major lower limb amputation.

- Participants had all undergone amputation due to complications of peripheral vascular disease; therefore findings may not be generalisable to patients undergoing amputation due to other factors such as trauma.

- While it was considered important to capture the views of patients at higher risk of postoperative delirium, this meant that some interview participants had poor understanding or recall of the trial intervention.

\section{INTRODUCTION}

Acute postoperative pain is common among amputees, ${ }^{1}$ and is associated with chronic residual and phantom limb pain. ${ }^{2}$ Long-term pain after amputation can have significant negative consequences, including depression, ${ }^{3}$ disability, ${ }^{4}$ unemployment ${ }^{5}$ and poorer health-related quality of life. ${ }^{6}$ Opioids are routinely used for pain management following lower limb amputation ${ }^{7}$; however, only a minority of lower limb amputees in the UK experience 'good' acute pain control. ${ }^{8}$ The side effects of opioids are well-established, including sedation, ${ }^{9}$ nausea and vomiting, ${ }^{10}$ constipation $^{11}$ and pruritus. ${ }^{9}$ Furthermore, opioid-related adverse drug events are associated with increased hospital costs and length of stay. ${ }^{12} 13$ Therefore, there is a need to examine the efficacy of alternative methods of analgesia following lower limb amputation.

There is evidence to suggest that placement of a perineural catheter (PNC) adjacent to a 
major nerve at the time of amputation, delivering a continuous infusion of local anaesthetic to the surgical site, may be effective in reducing acute pain following major lower limb amputation (amputation at a level above the ankle joint). A review and meta-analyses ${ }^{14} 15$ showed opioid consumption was significantly lower among patients receiving a PNC following amputation than among controls. However, the authors noted that the quality of available evidence was low, indicating a need for a randomised controlled trial to fully establish the efficacy of the PNC in reducing acute pain. In recognition of this, the PLACEMENT (Perineural Local Anaesthetic Catheter aftEr Major lowEr limb amputatioN Trial) trial was conducted. PLACEMENT was a randomised controlled feasibility trial comparing the use of a PNC versus standard care for postoperative pain relief following major lower limb amputation. ${ }^{16} 17$

It is believed no studies to date have focused on the qualitative experiences of patients receiving a PNC to relieve postoperative pain. Research suggests patient beliefs and attitudes towards pain management can influence key clinical outcomes ${ }^{18}$ highlighting the importance of exploring patient acceptance of analgesia. Attempting to capture experiences of pain using unidimensional measures is identified as a key challenge in clinical trials, ${ }^{19}$ as pain is a complex and subjective construct perceived diversely by individuals. ${ }^{20}$ Therefore, qualitative exploration can provide insights that may be missed if exclusively quantitative measures such as pain rating scales are used. The potential value of qualitative research in clinical trials is increasingly acknowledged, for example, in improving validity of trial measures, explaining outcomes and contextualising findings. ${ }^{21}$

Given the subjective nature of pain, and known clinical significance of patient perceptions of pain management, a qualitative work package - which would allow for in-depth exploration of patient experiences and complement quantitative outcome measures/analyses-was considered an essential component of the PLACEMENT trial design. The primary aim of the qualitative study was to explore patient experiences, understanding and perceptions of analgesia use following amputation. Specifically, we aimed to explore experiences of (1) opioids and (2) the PNC.

\section{METHODS}

\section{Study setting: the PLACEMENT trial}

This qualitative interview study was conducted as part of PLACEMENT - a randomised controlled feasibility trial. PLACEMENT was conducted with adult patients undergoing major lower limb amputation (above or below knee) due to complications of peripheral vascular disease, at two hospitals in South Wales in 2017. ${ }^{17}$ See the PLACEMENT protocol ${ }^{16}$ for full trial inclusion and exclusion criteria. The aim of the trial was to investigate the feasibility of conducting a full randomised controlled trial comparing the use of a PNC versus standard care for postoperative pain relief following amputation. Eligible patients were randomised preoperatively or intraoperatively to either the PNC or usual care group. Participants in both groups received standard postoperative analgesia, tailored to their individual needs. Those randomised to the PNC group received usual care plus local anaesthetic delivered via a PNC placed adjacent to a major nerve at the time of amputation, continued for up to 5 days. Participants were not informed of the group they were randomised to until the postoperative period. Pain data were recorded preoperatively and up to 6 months postoperatively (see the PLACEMENT protocol ${ }^{16}$ for full details of trial outcome measures).

Fifty patients were randomised, of which 49 completed 5-day follow-up.

\section{Patient and public involvement}

A discussion group attended by amputees was held prior to the PLACEMENT trial, to explore their experiences of postoperative pain relief and the acceptability of a trial examining PNC usage. Two patient and public involvement (PPI) representatives (one who had undergone major lower limb amputation, the other a relative of an amputee) contributed to trial development and management meetings, giving constructive feedback on trial processes and the embedded qualitative study. They also provided feedback on study documents (including participant information sheets and interview topic guides) and dissemination materials. They were involved in publicising main trial results, with one representative discussing findings on BBC Radio Wales.

\section{Participants and recruitment}

PLACEMENT participants who completed 5-day postoperative follow-up, were able and willing to participate in a face-to-face interview, and had consented to be contacted in relation to this, were eligible to take part in the qualitative study. Interview participants were purposively sampled to ensure representation of both treatment groups (PNC and usual care) and recruiting sites. To facilitate exploration of a range of patient experiences, we aimed to select a diverse sample in terms of age, gender and amputation type (above or below knee). Eligible patients were identified by research nurses and clinical teams at both sites; they were approached after the 5-day postoperative period and were provided with an information sheet explaining the qualitative study. Once patients had had sufficient time to read the study information, ask questions and consider whether they wanted to participate, they were invited to take part in an interview. Written informed consent was obtained from those who agreed to participate, and a mutually convenient time for the interview was arranged in liaison with a qualitative researcher.

To enable exploration of acute and long-term postoperative pain, interviews were conducted at two time points: time 1-within the postoperative period (up to 1 month following amputation) and time 2-at least 6 months following amputation. Patients who took part 
in interviews at time 1 were subsequently telephoned by a qualitative researcher and invited to take part in an interview at time 2 . Where participants were deceased or could not be contacted at time 2, patients with similar characteristics were identified from the PLACEMENT study database and telephoned by a member of the clinical team. If they verbally agreed to be contacted by a qualitative researcher, they were contacted and invited to take part in an interview. Written informed consent was obtained by the researcher prior to the commencement of each interview.

\section{Data collection}

Interviews were semi-structured with a set of open-ended questions and prompts used to guide discussion. Interview topic guides were developed in consultation with clinician and PPI representative members of the PLACEMENT Trial Management Group. Time 1 interviews opened with questions about participant experiences of pain prior and leading up to amputation (eg, type of pain, severity, impact on quality of life), and moved on to discuss participant experiences of pain after amputation and postoperative pain management, including need for analgesia, side effects and perceptions of different modes of analgesia. Time 2 interviews opened with general questions about participants' recovery since amputation, followed by exploration of pain experienced (eg, stump pain or phantom limb pain), changes in pain and pain management, including perceptions of the PNC. The interview topic guide was reviewed following the time 1 interviews, and new questions added to explore previously unanticipated themes. For example, at time 1, some participants described experiencing considerable variation in pain at different times of day. Therefore, in the time 2 interviews, participants were specifically asked whether they experienced pain at particular times, such as getting up in the morning, or at night.

Time 1 interviews were conducted between May and October 2017; time 2 interviews were conducted between April and July 2018. To maximise accessibility, interviews took place in participants' own homes or in a private room in hospital if participants were inpatients. For one interview at time 1 and three interviews at time 2 , the spouse or partner of the participant was present. Interviews were conducted by SM, HS and MM-all experienced qualitative health researchers who had no involvement in main trial procedures and were not previously known to participants. The interviewers made field notes during and/ or after the interviews to record contextual information and initial thoughts about research themes. Interviews were audio-recorded and professionally transcribed then anonymised. Verbatim transcripts were quality-checked and imported into NVivo (V.11) for analysis.

\section{Data analysis}

A systematic framework approach to analysis was taken, enabling exploration of the full dataset while facilitating comparison within and between cases. Analysis was

\begin{tabular}{lc}
\hline Table 1 Interview participant characteristics \\
\hline Characteristic & $\mathbf{n}$ \\
\hline Gender & \\
Male & 10 \\
Female & 4 \\
Age range (years) & \\
46-55 & 3 \\
56-65 & 1 \\
66-75 & 5 \\
76-85 & 4 \\
86-95 & 1 \\
Amputation type & \\
Below knee & 9 \\
Above knee & 5 \\
Trial arm & \\
PNC & 7 \\
Usual care & 7 \\
Interview participation & 4 \\
Both interviews & 4 \\
Time 1 interview only & \\
Time 2 interview only & \\
\hline
\end{tabular}

PNC, perineural catheter.

primarily conducted by SM, with regular meetings held throughout the analytic process between SM, HS and LB-H to discuss and refine emerging themes. There were five stages of analysis. ${ }^{22}$ First, all interview transcripts and field notes were read in full and key themes and ideas noted. Second, a thematic framework was constructed, informed deductively by the research aim and interview questions, and inductively by issues raised by participants. This enabled a focused approach to analysis, while allowing for the generation of unanticipated themes from the data. Third, each transcript was coded by SM (using NVivo V.11) in accordance with the thematic framework. To enhance reliability and validity, 4 of the 14 transcripts were double coded by LB-H. Discrepancies were discussed, which allowed for refinement of the coding framework and further development of research themes. Fourth, data were organised thematically into tables, providing a visual representation of the whole dataset. Finally, these thematic tables were used to identify key dimensions of the data in relation to the research question. We used the Consolidated criteria for Reporting Qualitative research checklist $^{23}$ in the reporting of interview findings.

\section{RESULTS}

Twenty interviews were conducted with 14 participants (see table 1). At the time data collection ceased, no new themes were emerging from the data, therefore saturation was reached. Interviews lasted between 24 and $109 \mathrm{~min}$ 


\begin{tabular}{ll}
\hline Table 2 Interview themes and subthemes \\
\hline Theme & Subtheme \\
\hline $\begin{array}{l}\text { Perceptions and } \\
\text { experiences of the PNC }\end{array}$ & $\begin{array}{l}\text { Valuing localised continuous delivery } \\
\text { Acceptability of the PNC }\end{array}$ \\
$\begin{array}{l}\text { Understanding of } \\
\text { postoperative analgesia }\end{array}$ & $\begin{array}{l}\text { Knowledge and understanding of } \\
\text { the PNC } \\
\text { Understanding of analgesia received }\end{array}$ \\
$\begin{array}{ll}\text { Perceptions and } \\
\text { experiences of opioid } \\
\text { analgesia }\end{array}$ & $\begin{array}{l}\text { Concerns regarding side effects } \\
\text { Experience of side effects }\end{array}$ \\
$\begin{array}{l}\text { Self-management of } \\
\text { postoperative analgesia }\end{array}$ & $\begin{array}{l}\text { Dependence-related concerns } \\
\text { Limiting pain relief }\end{array}$ \\
\hline
\end{tabular}

PNC, perineural catheter.

(mean 55.3). Of the 10 participants interviewed at time 1 , 6 took part in a second interview. The remaining 4 participants were deceased $(\mathrm{N}=2)$ or could not be contacted $(\mathrm{N}=2)$ and were replaced with new participants matched as closely as possible in terms of trial arm, age, gender and amputation type. Interview participants were broadly similar to the overall PLACEMENT trial population in terms of gender ( $71 \%$ male vs $80 \%$ male), age (mean 70 vs mean 70$)$ and amputation type ( $64 \%$ below knee vs $61 \%$ below knee).

Four key themes were discussed by participants, each with two subthemes (see table 2): (1) perceptions and experiences of the PNC; (2) understanding of postoperative analgesia; (3) perceptions and experiences of opioid analgesia; (4) self-management of postoperative analgesia. Participant quotes used to illustrate findings are labelled with a participant ID number, trial group (PNC or control), and interview time point (time 1 or time 2 ).

\section{Perceptions and experiences of the PNC}

Valuing localised, continuous delivery

Participants who received the PNC tended to value the localised, direct and continuous nature of this mode of analgesia, for example, highlighting the benefit of having pain relief 'on tap' (see table 3).

\section{Acceptability of the PNC}

Those in the PNC group appeared to find the placement of the PNC acceptable in practical terms, with some reporting they did not notice its presence. No side effects were reported. One control group participant explained they would have felt reassured prior to their amputation had they been told they would receive the PNC.

\section{Understanding of postoperative analgesia \\ Knowledge and understanding of the PNC}

A minority of participants demonstrated a good understanding of how the PNC delivered pain relief (see table 4). However, at the time of interview most participants did not know or recall that the PNC delivered local anaesthetic. There appeared to be confusion between the $\mathrm{PNC}$ and opioid or patient-controlled analgesia.

\section{Understanding of analgesia received}

Participants in both groups tended to have a poor understanding or recall as to whether they received the PNC. Some did not know if they had received the PNC, while others believed they received the PNC although they were in the control group, and vice versa. Most participants in both groups reported the pain relief they received was highly effective.

\section{Perceptions and experiences of opioid analgesia \\ Concerns regarding side effects}

Participants in both groups expressed concerns about the potential side effects of opioid analgesia, including addiction and withdrawal symptoms (see table 5 ).

\section{Experience of side effects}

Participants reported experiencing several side effects of opioids, including confusion, nausea, vomiting, drowsiness and hallucinations. Reporting of analgesic side

\begin{tabular}{ll}
\hline Table 3 & Perceptions and experiences of the PNC \\
\hline Subtheme & Illustrative quotes \\
\hline $\begin{array}{l}\text { Valuing } \\
\text { localised, } \\
\text { continuous } \\
\text { delivery }\end{array}$ & $\begin{array}{l}\text { Participant 210, PNC group, Time 1: The catheter is really, really good because every time there's [pain relief] going into } \\
\text { Participant 116, PNC group, Time 1: They put that thing down to the nerve ending and ... by ten o'clock that night it'd } \\
\text { kicked in, ooh did it kick in. }\end{array}$ \\
& $\begin{array}{l}\text { Participant 230, PNC group, Time 2: Hand on heart, with that thing on my leg, I had no pain at all ... I would } \\
\text { recommend it to anybody. }\end{array}$ \\
$\begin{array}{l}\text { Acceptability } \\
\text { of the PNC }\end{array}$ & $\begin{array}{l}\text { Participant 105, PNC group, Time 2: It was feeding me drugs you know ... They put it on, like, you know, I just forgot } \\
\text { about it. }\end{array}$ \\
& $\begin{array}{l}\text { Participant 116, PNC group, Time 2: Apart from the anaesthetist having to come every night to [refill it] ... it wasn't a } \\
\text { Problem. }\end{array}$ \\
& $\begin{array}{l}\text { Participant 110, Control group, Time 2: I was hoping, to be honest with you, that I would have been [in the PNC group] } \\
\text { not knowing whether or not you have the [local] anaesthetic ... but I think it's anticipatory pain isn't it sometimes? You } \\
\text { think, oh you've had your leg off, it's going to be awful, painful and all this. }\end{array}$ \\
\hline
\end{tabular}

PNC, perineural catheter. 
Table 4 Understanding of postoperative analgesia

\begin{tabular}{|c|c|}
\hline Subtheme & Illustrative quotes \\
\hline $\begin{array}{l}\text { Knowledge and } \\
\text { understanding } \\
\text { of the PNC }\end{array}$ & $\begin{array}{l}\text { Participant 116, PNC group, Time 2: [I had a] thing they put on filled with local anaesthetic. That was going right into } \\
\text { the wound ... and then this stuff just fed into it over a 24-hour period. } \\
\text { Participant } 210, \text { PNC group, Time 1: [The PNC] was good because it was releasing morphine ... into your stump. } \\
\text { Participant 107, Control group, Time 1: [The PNC is] supposed to have been a ball put inside, before they close it up. } \\
\text { And every time you get a pain, you should have a button and you press it. Right? And that sends morphine out. }\end{array}$ \\
\hline $\begin{array}{l}\text { Understanding } \\
\text { of analgesia } \\
\text { received }\end{array}$ & $\begin{array}{l}\text { Participant 109, Control group, Time 1: [The PNC] was wonderful you know. I recommend it. In, in the future, they } \\
\text { [could] do away with the injections and tablets you know. They can give it to you straightaway ... It's in your system } \\
\text { then isn't it, no more waiting ... I didn't have no pain with the ... you know, with what they're doing. } \\
\text { Participant 107, Control group, Time 1: [The pain relief] went ... straight into the wound ... straight to the spot .... the } \\
\text { pain went straightaway. } \\
\text { Participant 115, Control group, Time 1: They might have put [the PNC] in for all I know, I don't know. } \\
\text { Participant 220, PNC group, Time 1: } \\
\text { Interviewer: So they didn't insert anything ... into the leg by the sounds of it? } \\
\text { Participant: No, not into the leg; into my arm. }\end{array}$ \\
\hline
\end{tabular}

PNC, perineural catheter.

effects appeared to be more prevalent in the control than the PNC group.

\section{Self-management of postoperative analgesia}

Dependence-related concerns

Participants discussed their own role in postoperative pain management, with some highlighting concerns about becoming dependent on opioids (see table 6).

\section{Limiting pain relief}

Most participants explained they had tried to limit their intake of analgesia following surgery-either in terms of dosage or in their choice of medication (eg, choosing more mild forms of analgesia, such as paracetamol)even when they experienced pain. Some described how they had tried to reduce their medication over time, citing concerns about side effects and potential dependence or overdose. One participant highlighted how the PNC may reduce anxiety associated with pain management. In contrast with patient-controlled analgesia, which some were concerned about using, the PNC relieved participants of responsibility for their analgesia and allowed them to receive pain relief automatically.

\section{DISCUSSION}

This is the first study to provide a qualitative insight into the experiences of patients receiving a PNC following major lower limb amputation. Findings highlight that continuous delivery of postoperative analgesia via a PNC may reduce the burden of pain management on patients. The study also advances understanding of patients' perceptions and experiences of analgesia, building on previous findings that dependence-related anxiety causes patients to self-limit pain relief.

Interview participants reported the PNC was effective in relieving postoperative pain. This supports quantitative findings that PNC use may significantly reduce opioid consumption. ${ }^{14} 15$ Participants particularly valued receiving localised, continuous analgesia, and found the placement of the PNC acceptable. The belief that localised pain relief was being administered also appeared to introduce a placebo effect: participants from both study arms who believed they had received a PNC reported relief from pain and/or pain-related anxiety. One participant suggested that if they had known they would receive the PNC in advance of their surgery, this would have reduced their preoperative anxiety. Given the well-documented

Table 5 Perceptions and experiences of opioid analgesia

\section{Sub-theme Illustrative quotes}

Concerns Participant 116, PNC group, Time 1: I can handle a fair amount of pain ... the side effects of painkillers are worse.

regarding Participant 223, Control group, Time 2: I do suffer effects when I give up these morphine tablets. You really get hooked on side effects them very quickly ... it's the most horrible sensation ... You literally can't keep still.

Experience Participant 104, Control group, Time 2: I was seeing things that weren't there ... I saw people behind the television, of side little miniature people, like monkeys ... I was worried to death, I thought l'd go mad ... I had the police here three times effects one day ... I could see the bloody, the wardrobe door opening ... Once I stopped taking that Oramorph, just a couple of days and it all finished. I've never had no bother since.

Participant 110, Control group, Time 2: I had a reaction to the morphine. My respirations went down and ... I was like all over the place, but so sick. I was sick constantly ... I was just feeling sick every time I moved or turned.

PNC, perineural catheter. 
Table 6 Self-management of postoperative analgesia

\begin{tabular}{ll}
\hline Subtheme & Illustrative quotes \\
\hline $\begin{array}{l}\text { Dependence- } \\
\text { related }\end{array}$ & Participant 109, Control group, Time 1: [If I'd had the morphine pump for longer]l think I [would] have got then too used \\
concerns & to it, you know, rely on it. You know, it's so easy to press a button. \\
& Participant 115, Control group, Time 1: I have Oramorph ... three times a day, and the first thing [I] ask is, is it habit \\
& forming? And they said well anything can be habit forming. \\
& Participant 108, PNC group, Time 2: I don't want to keep on taking tablets... You can get used to taking tablets for \\
& nothing, really ... And you perhaps shouldn't get used to them, you should fight the pain off before you take tablets. \\
Limiting pain & Participant 107, Control group, Time 1: I've got to put up with [the pain] and that's it ... I don't ask the nurse [for pain \\
relief & relief unless] I'm in agony. \\
& Participant 108, PNC group, Time 1: I could do with [pain relief] sometimes but I don't know why, I try not to you know \\
& have too much medicine. \\
& Participant 220, PNC group, Time 1: I tend to stick to the Paracetamol. \\
& Participant 109, Control group, Time 1: I weaned myself off [Oramorph, then] I thought now I've got to cut these Co- \\
& codamols out. I thought, I might be overdosing myself. I'm only having eight a day on some days you know, if I cut \\
& them down, and go on Paracetamol now, and touch wood again, I feel alright. \\
& Participant 223, Control group, Time 2: I don't like taking [morphine] and I've asked the doctor and dropped to five \\
& milligrams ... I'm trying to get back to just Paracetamol. \\
& Participant 107, Control group, Time 1: If I'm desperate for pain relief, right, I know I shouldn't have more Oramorph \\
& because it ... affects the brain sometimes ... But I only have 3 mil [sic] ... Some of them have 5 mil, but I don't ... \\
& Because 3 mil is enough. But some people have it morning and night, when it's no good to you. \\
& Participant 210, PNC group, Time 1: I found [the PNC] good because I didn't have to press nothing ... you don't \\
& have to fiddle because you can't overdose yourself, and every time there's [pain relief] going into your stump and that \\
doesn't hurt then.
\end{tabular}

PNC, perineural catheter.

side effects of opioids, ${ }^{9-13}$ these findings suggest routine placement of a PNC following amputation could improve both the preoperative and postoperative experience of patients.

Despite receiving both written and verbal information relating to the PNC preoperatively, participants appeared to have a poor understanding of the type of postoperative analgesia they received. Limited recall of preoperative information following surgery is highlighted in a range of patient populations. ${ }^{24-26}$ Postoperative delirium is comparatively common among vascular and older patients $^{27} 28$ and participants in this study generally demonstrated poor recall of administered analgesia; a factor likely to have affected comprehension. A lack of understanding of postoperative pain control is detrimental to patient outcomes. For example, in one survey, ${ }^{29}$ participants who had difficulty using patient-controlled analgesia (over a quarter of the sample) reported lower satisfaction and were significantly less likely to feel they could control their pain. Given that the PNC delivers continuous analgesia and does not rely on patients' ability to express their need for pain relief or effectively use patient-controlled analgesia, it is likely to particularly benefit populations at greater risk of postoperative delirium.

Consistent with previous research, ${ }^{30-32}$ participants reported concerns about becoming dependent on pain relief medication, and explained this had caused them to limit their immediate intake of analgesics and/or attempt to reduce this over time. Patient anxiety may result in inadequate pain management, ${ }^{33}$ so it is important that such concerns are identified and addressed by clinicians. Given the incidence of self-limiting of pain medication, use of a continuous analgesic such as that delivered via a PNC may be effective in reducing postoperative pain. This delivery method has the potential to reduce patient anxieties relating to overdose and addiction, together with the psychological burden of restricting pain relief. It could also help overcome the reluctance of some patients to express concerns relating to their own pain or to request analgesia. It is suggested ${ }^{34}$ that patients may not value being in control of their own analgesia; therefore there is a clear need for future research exploring patient anxieties and satisfaction related to pain management, comparing continuous analgesia with patientadministered pain medication.

Study findings should be considered in the context of the following limitations. First, all interview participants were undergoing amputation due to complications of peripheral vascular disease. We considered it important to focus on this population because of the unique challenges posed in terms of postoperative pain management, for example, the common contraindication of epidural anaesthesia for this group, ${ }^{35}$ mainly due to concomitant use of antithrombotic medication. However, findings are not generalisable to patients undergoing amputation resulting from other factors such as trauma. Second, as participants were interviewed by members of the PLACEMENT team, they may have felt obliged to report positive experiences of the PNC. To mitigate this, interviewers had no involvement in trial delivery, and it was emphasised at the start of each interview that there were no right or wrong answers and responses would be anonymous. Third, interview participants appeared to have a poor understanding of the trial intervention, meaning some could not accurately comment on their experiences of 
receiving a PNC. Postoperative delirium is recognised as a particular issue among the patient group under study; nonetheless it was considered important to capture the views of those most likely to benefit from improvements to postoperative pain management. Conducting a second set of interviews at least 6 months following amputation enabled discussion with participants once they had more fully recovered from surgery. While this may have introduced issues with recall, participants appeared able to vividly recollect their experiences. As PLACEMENT was a feasibility trial, in the qualitative study for the full trial it is proposed to conduct interviews 1-2 months following amputation, aiming to limit the impact of both postoperative delirium and recall issues.

Although including control group participants allowed the exploration of feelings relating to not receiving the PNC and understanding of the PNC among those who did not receive it, direct experiences of the PNC could not be captured from this group. While experiences of the PNC itself could not be fully explored in all interviews, participants provided in-depth descriptions of their experiences and anxieties relating to opioids and self-management of pain medication, highlighting the potential benefit of continuous rather than patient-controlled analgesia. In the qualitative study for the full PLACEMENT trial it is proposed to conduct a greater proportion of interviews with participants in the PNC than in the control group.

Findings have clear implications for clinical practice. Placement of a PNC following major lower limb amputation could benefit patients by alleviating the burden of pain management, reducing postoperative pain and preoperative anxiety, and decreasing opioid use. The delivery of continuous analgesia may particularly benefit populations at greater risk of postoperative delirium and those who are reluctant to request pain relief. More generally, this study highlights the need for effective alternatives to patient-controlled analgesia, which can result in anxiety and inadequate pain management. Future research could further explore patient anxieties and satisfaction related to different modes of analgesia, for example, comparing continuous analgesia with patientadministered pain medication.

\section{CONCLUSION}

Unanticipated benefits of PNC usage for postoperative pain were identified, including the potential to reduce the burden of pain management on patients. Insights such as these may be overlooked in traditional quantitative studies, emphasising the value of qualitative approaches to surgical research. Findings suggest routine placement of a PNC following amputation could improve pain management, particularly for patient groups at risk of postoperative delirium. Future research could further examine the comparison between patient-controlled analgesia and continuous analgesia, in relation to patient anxiety and satisfaction with pain management. Exploring the efficacy of PNC analgesia in other patient groups, such as those undergoing trauma-related lower limb amputation, would indicate whether findings are more broadly applicable.

Acknowledgements We thank all the patients who gave up their time to participate in interviews, and all members of the PLACEMENT study team. This work was supported by the Welsh Government through Health and Care Research Wales, via the Research for Patient and Public Benefit (RfPPB) scheme [reference number 1198]. The study was sponsored by Aneurin Bevan University Health Board Newport, South Wales.

Contributors CPT, DCB and GKA led the development of the PLACEMENT trial design, grant application and implementation of the trial protocol, together with ET-J and LB-H. C-AW was the trial manager and ET-J the senior trial manager who coordinated the operational delivery of the study protocol and recruitment. LB-H designed and coordinated the qualitative study. SM, HS and MM conducted patient interviews, and SM, HS and LB-H conducted the qualitative analysis. DH was responsible for data management. SM prepared the first draft of the manuscript. All authors provided critical review and final approval of the manuscript. SM is the author acting as guarantor for the overall content of this article.

Funding This work was supported by the Research for Patient and Public Benefit (RfPPB) programme, Health and Care Research Wales, reference number 1198. The Centre for Trials Research receives funding from Health and Care Research Wales and Cancer Research UK.

Competing interests None declared.

Patient and public involvement Patients and/or the public were involved in the design, or conduct, or reporting, or dissemination plans of this research. Refer to the Methods section for further details.

Patient consent for publication Not applicable.

Ethics approval Written informed consent was obtained from all study participants. The main PLACEMENT trial and this interview study were approved by Wales Research Ethics Committee 3 (reference number 16/WA/0353) and were conducted in accordance with the principles of the Declaration of Helsinki.

Provenance and peer review Not commissioned; externally peer reviewed.

Data availability statement No data are available. The datasets generated and analysed for the study are not publicly available and cannot be shared as individual privacy could be compromised if full interview transcripts were released.

Open access This is an open access article distributed in accordance with the Creative Commons Attribution Non Commercial (CC BY-NC 4.0) license, which permits others to distribute, remix, adapt, build upon this work non-commercially, and license their derivative works on different terms, provided the original work is properly cited, appropriate credit is given, any changes made indicated, and the use is non-commercial. See: http://creativecommons.org/licenses/by-nc/4.0/.

\section{ORCID iDs}

Sarah Milosevic http://orcid.org/0000-0003-1973-8286

Cherry-Ann Waldron http://orcid.org/0000-0001-8465-2492

\section{REFERENCES}

1 Suer M, Sehgal N. Post-amputation pain. In: Suer M, Sehgal N, eds. Questions and answers in pain medicine. Cham: Springer, 2021: 143-7.

2 Larbig W, Andoh J, Huse E, et al. Pre- and postoperative predictors of phantom limb pain. Neurosci Lett 2019;702:44-50.

3 Roepke AM, Turner AP, Henderson AW, et al. A prospective longitudinal study of trajectories of depressive symptoms after dysvascular amputation. Arch Phys Med Rehabil 2019;100:1426-33.

4 Morgan SJ, Friedly JL, Amtmann D, et al. Cross-sectional assessment of factors related to pain intensity and pain interference in lower limb prosthesis users. Arch Phys Med Rehabil 2017;98:105-13.

5 Schoppen T, Boonstra A, Groothoff JW, et al. Factors related to successful job reintegration of people with a lower limb amputation. Arch Phys Med Rehabil 2001;82:1425-31.

6 Torbjörnsson E, Ottosson C, Boström L, et al. Health-related quality of life and prosthesis use among patients amputated due to peripheral arterial disease - a one-year follow-up. Disabil Rehabil 2020:1-9.

7 Neil MJE. Pain after amputation. BJA Educ 2016;16:107-12. 
8 Gough MJ, Juniper M, Freeth H. Lower limb amputation: working together. London: National Confidential Enquiry into Patient Outcome and Death, 2014.

9 Richman JM, Liu SS, Courpas G, et al. Does continuous peripheral nerve block provide superior pain control to opioids? A metaanalysis. Anesth Analg 2006;102:248-57.

10 de Boer HD, Detriche O, Forget P. Opioid-related side effects: postoperative ileus, urinary retention, nausea and vomiting, and shivering. A review of the literature. Best Pract Res Clin Anaesthesiol 2017;31:499-504.

11 Miaskowski C. A review of the incidence, causes, consequences, and management of gastrointestinal effects associated with postoperative opioid administration. J Perianesth Nurs 2009;24:222-8.

12 Oderda GM, Said Q, Evans RS, et al. Opioid-related adverse drug events in surgical hospitalizations: impact on costs and length of stay. Ann Pharmacother 2007;41:400-7.

13 Gan TJ. Poorly controlled postoperative pain: prevalence, consequences, and prevention. J Pain Res 2017;10:2287-98.

14 Bosanquet DC, Glasbey JCD, Stimpson A, et al. Systematic review and meta-analysis of the efficacy of perineural local anaesthetic catheters after major lower limb amputation. Eur J Vasc Endovasc Surg 2015;50:241-9.

15 Laloo R, Ambler GK, Locker D, et al. Systematic review and metaanalysis of the effect of perineural catheters in major lower limb amputations. Eur J Vasc Endovasc Surg 2021;62:295-303.

16 Bosanquet DC, Ambler GK, Waldron C-A, et al. Perineural local anaesthetic catheter after major lower limb amputation trial (PLACEMENT): study protocol for a randomised controlled pilot study. Trials 2017;18:629.

17 Bosanquet DC, Ambler GK, Waldron C-A, et al. Perineural local anaesthetic catheter after major lower limb amputation trial (PLACEMENT): results from a randomised controlled feasibility trial. BMJ Open 2019;9:e029233.

18 Linde K, Witt CM, Streng A, et al. The impact of patient expectations on outcomes in four randomized controlled trials of acupuncture in patients with chronic pain. Pain 2007;128:264-71.

19 Gilron I, Carr DB, Desjardins PJ, et al. Current methods and challenges for acute pain clinical trials. Pain Rep 2019;4:e647.

20 van Dijk JFM, van Wijck AJM, Kappen TH, et al. Postoperative pain assessment based on numeric ratings is not the same for patients and professionals: a cross-sectional study. Int J Nurs Stud 2012;49:65-71.
21 O'Cathain A, Thomas KJ, Drabble SJ, et al. What can qualitative research do for randomised controlled trials? A systematic mapping review. BMJ Open 2013;3:e002889.

22 Ritchie J, Spencer L. Qualitative data analysis for applied policy research. In: Bryman A, Burgess R, eds. Analyzing qualitative data. London: Routledge, 2002: 187-208.

23 Tong A, Sainsbury P, Craig J. Consolidated criteria for reporting qualitative research (COREQ): a 32-item checklist for interviews and focus groups. Int J Qual Health Care 2007;19:349-57.

24 Crepeau AE, McKinney BI, Fox-Ryvicker M, et al. Prospective evaluation of patient comprehension of informed consent. J Bone Joint Surg Am 2011;93:e114.

25 Gillies A, Gillies R, Weinberg L. Patient recollections of perioperative anaesthesia risks. Anaesth Intensive Care 2013;41:247-50.

26 Uzzaman MM, Sinha S, Shaygi B, et al. Evaluation of patient's understanding and recall of the consent process after open inguinal hernia repairs. Int J Surg 2012;10:5-10.

27 Balasundaram B, Holmes J. Delirium in vascular surgery. Eur $J$ Vasc Endovasc Surg 2007;34:131-4.

28 Visser L, Prent A, van der Laan MJ, et al. Predicting postoperative delirium after vascular surgical procedures. J Vasc Surg 2015;62:183-9.

29 Patak LS, Tait AR, Mirafzali L, et al. Patient perspectives of patientcontrolled analgesia (PCA) and methods for improving pain control and patient satisfaction. Reg Anesth Pain Med 2013;38:326-33.

30 Chumbley GM, Hall GM, Salmon P. Patient-controlled analgesia: an assessment by 200 patients. Anaesthesia 1998;53:216-21.

31 Conrardy M, Lank P, Cameron KA, et al. Emergency department patient perspectives on the risk of addiction to prescription opioids. Pain Med 2016;17:114-21.

32 Lewis ET, Combs A, Trafton JA. Reasons for under-use of prescribed opioid medications by patients in pain. Pain Med 2010;11:861-71.

33 Wilder-Smith $\mathrm{CH}$, Schuler L. Postoperative analgesia: pain by choice? The influence of patient attitudes and patient education. Pain 1992;50:257-62.

34 Taylor NM, Hall GM, Salmon P. Patients' experiences of patientcontrolled analgesia. Anaesthesia 1996;51:525-8.

35 Nikolajsen L, Ilkjaer S, Christensen JH, et al. Randomised trial of epidural bupivacaine and morphine in prevention of stump and phantom pain in lower-limb amputation. Lancet 1997;350:1353-7. 